Cipango Cahiers d'études japonaises

$18 \mid 2011$

Le Japon et le fait colonial I

\title{
Le Genji monogatari : un roman en wabun
}

À propos de l'ouvrage Le Roman du Genji : opacité et transparence, Matsui Kenji, Université de Komazawa

Sumie Terada et Kenji Matsui

Traducteur : Catherine Garnier

(2) OpenEdition

Journals

Édition électronique

URL : https://journals.openedition.org/cipango/1596

DOI : 10.4000/cipango.1596

ISSN : 2260-7706

Éditeur

INALCO

Édition imprimée

Date de publication : 1 juin 2011

Pagination : 237-259

ISBN : 978-2-85831-195-8

ISSN : $1164-5857$

Référence électronique

Sumie Terada et Kenji Matsui, « Le Genji monogatari : un roman en wabun », Cipango [En ligne], 18 |

2011, mis en ligne le 23 mars 2013, consulté le 30 juin 2021. URL : http://journals.openedition.org/ cipango/1596; DOI : https://doi.org/10.4000/cipango.1596

Ce document a été généré automatiquement le 30 juin 2021.

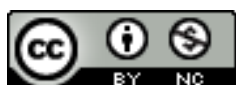

Cipango est mis à disposition selon les termes de la Licence Creative Commons Attribution - Pas d'Utilisation Commerciale 4.0 International. 


\title{
Le Genji monogatari : un roman en wabun
}

À propos de l'ouvrage Le Roman du Genji : opacité et transparence, Matsui Kenji, Université de Komazawa

\author{
Sumie Terada et Kenji Matsui
}

Traduction : Catherine Garnier

\section{NOTE DE L'ÉDITEUR}

FUJIWARA Katsumi, TAKADA Hirohiko et TERADA Sumie (dir.), 2008nen Pari sinpojiumu : Genji monogatari no tōmeisa to futōmeisa-bamen, waka, katari, jikan no bunseki o tooshite 200 8 年パリシンポジウム：源氏物語の透明さと不透明さ場面・和歌・語り・時間の分析を 通して一(Colloque international franco-japonais sur Le Roman du Genji : Opacité et transparence - scènes, langage poétique, temps et narration -), Tōkyō, 2009, Seikansha, 233 p.

\section{Présentation par Terada Sumie}

1 Les années 2008 et 2009 représentent une période tourmentée pour les enseignants - chercheurs français, et la présentation du livre Le Roman du Genji : opacité et transparence - scènes, langage poétique, temps et narration reflète cette tranche de l'histoire qui a une lourde signification pour beaucoup d'entre nous. Dans son discours d'ouverture du colloque qui a eu lieu en mars 2008, Anne Bayard-Sakai oppose le travail à long terme de notre équipe, centré sur Le Roman du Genji, à "la politique scientifique qui ne reconnaît que la vision à court terme». Fujiwara Katsumi, qui tenait à mettre ce discours en guise de préface et à intégrer la date du colloque dans le titre de l'ouvrage, se réfère dans sa synthèse des articles à La princesse de Clèves. En effet, cette œuvre avait été portée par les universitaires pendant cette période comme une figure symbolisant le mouvement d'opposition à une politique scientifique visant à soumettre la recherche et l'enseignement aux lois du marché. Durant les manifestations qui se sont enchaînées pendant cette période de lutte commencée en février 2009, nous nous sommes 
interrogés nous-mêmes sur le sens de notre travail qui s'organise autour du Roman du Genji, œuvre que nous nous plaisions à comparer à La Princesse de Clèves.

Dans ce contexte, il nous a paru que la lecture extrêmement lente imposée par la traduction collective avait une valeur salutaire, dans le sens où elle nous permettait de nous approcher des réalités d'un texte apprécié et transmis pendant plus de mille ans, et cela-est-ce illusion de notre part? - avec une intensité proportionnelle au temps que nous mettions à traduire. Accepter tout simplement de mettre autant de temps qu'il faut afin d'établir un passage entre deux langues aussi éloignées que le sont le français moderne et le japonais ancien, est apparu comme une méthode en soi, dans la situation de recherche que nous vivons actuellement et dans une société où les opérations qui s'effectuent en un millième de seconde font gagner des millions d'euros. Nous avons expliqué cette position dans la postface, et c'est dans cet esprit, que nous avons préparé la publication du colloque de 2008 centré sur le texte et son épaisseur.

3 Le livre lui-même, reprenant l'organisation du colloque, s'articule autour de trois sessions. La première (scènes, regards, espace théâtral) est composée, dans l'ordre de présentation, des articles de Daniel Struve (vision à la dérobée), de Kawazoe Fusae (Le Roman du Genji et le nō) et de Sano Midori (Le Roman du Genji et les peintures). La deuxième (la narration et le langage poétique) rassemble les articles de Terada Sumie (le brouillard en tant que motif narratif), de Hijikata Yōichi (le waka et la voix collective) et de Jacqueline Pigeot (le waka, dialogue et citation). La troisième session (le temps et la narration) est composée des écrits d'Anne BayardSakai (les anecdotes, le lecteur et la relecture), de Takada Hirohiko (le lieu privilégié et la formation du roman) et de Fujiwara Katsumi (l'ironie et le romanesque).

4 Nous avons demandé à Matsui Kenji, un des meilleurs spécialistes actuels dans le domaine de la littérature classique japonaise (période de Heian, notamment Le Roman du Genji) une note de lecture libre, qui consiste, au lieu de faire une recension, à développer les réflexions que lui a inspiré la lecture du livre. Autrement dit, nous avons souhaité provoquer une réaction au lieu d'un compte-rendu et d'un jugement. Il a ainsi librement choisi les articles qui entraient dans le domaine de son intérêt actuel. Pour terminer ces quelques précisions sur le cadre de son essai, il nous reste donc à présenter trois articles, ceux de Kawazoe Fusae, de Sano Midori et de Fujiwara Katsumi que Matsui n'a pas traités.

5 Le Roman du Genji est né dans une tradition de l'écriture fragmentaire. Réaliser une œuvre de fiction longue et solidement construite sur le plan narratif était à l'époque un exploit. Kawazoe Fusae et Takada Hirohiko abordent cette question. Takada l'étudie en se focalisant sur l'évolution, au cours de la rédaction, du rôle d'une résidence, Rokujō-in, lieu éminemment symbolique représentant le faste et le pouvoir du Genji. Si Takada essaie donc de cerner cette question qui était à l'œuvre au moment de la création, Kawazoe prend une direction opposée: elle compare le chapitre "L'arbre sacré (賢木 Sakaki) » à la pièce de nō du XV siècle inspirée de cet épisode et de son héroïne Rokujō ("Le Roman du Genji et la dramaturgie du nō inspiré de ce roman: comparaison avec Le temple de la lande No no miya 野々宮»). Sans se contenter d'une simple étude de réception, orientation fleurissante ces dernières années, elle utilise l'écart entre ces deux œuvres séparées par trois siècles et de genres différents (roman et théâtre) afin de dégager la spécificité de l'écriture romanesque créé par Murasaki Shikibu. Après une analyse fine du passage introductif du chapitre décrivant un paysage où alternent les points de vue du héros et de la narratrice, qu'elle considère comme une étape indispensable pour les retrouvailles apaisées des amants, elle souligne, à travers la comparaison avec la pièce du nō, la complexité du texte du Roman du Genji tissé d'innombrables points de vue à la fois explicites et implicites. Et elle démontre la différence de conception "narrative » entre ces deux œuvres en s'intéressant au traitement contrasté d'un objet, une bandelette votive (木綿 yū). Dans l'univers de la 
subjectivité pure du nō qui s'organise, dit-elle, autour du point de vue du personnage principal, cet objet religieux incarnant le destin de l'héroïne s'en va emporté par le courant d'une rivière. Par contre, dans Le Roman du Genji cet objet que le Genji offre à sa fille présage le développement d'un nouvel épisode qui se formera autour de ces deux personnages. Cet exemple illustre en effet une maturité étonnante de l'art romanesque chez Murasaki Shikibu, et prouve la pertinence de cette approche comparative. L'article incite également à nous interroger sur les différents types de subjectivité que la littérature japonaise a développés au cours de son histoire.

Sano Midori, dans son article "Forme du souvenir et souvenirs de la forme - Le Roman du Genji et la peinture", pose la question des images que suscite ce roman. Comme Kawazoe, sans se contenter d'une simple étude de réception, elle pose les problématiques des images dans un rapport dynamique entre le roman et les cuvres picturales: elle scrute d'abord le potentiel pictural que renferme le roman (la conception de la peinture énoncée dans différents passages et les citations de tableaux connus), puis la possibilité de la relecture de l'univers romanesque qu'offrent les œuvres picturales. Les paravents qu'elle choisit comme principaux supports de son analyse illustrent avec netteté les effets narratifs que les images produisent grâce aux assemblages et aux combinaisons contrastées des scènes, d'une part, et, d'autre part, par les jeux de détails qui fonctionnent comme citations des épisodes insérés dans d'autres épisodes. Elle nous présente ainsi le mécanisme dynamique où se perpétue l'interaction entre les mémoires romanesques que véhiculent les images, et les souvenirs des formes picturales qui recomposent la lecture du roman. Elle s'intéresse comme Kawazoe à un élément de détail qui joue un rôle important dans la construction "narrative», un jeune cerisier (wakagi no sakura 若木の桜) inséré dans un paysage d'un paravent, qui fonctionne comme repère d'un épisode dans le chapitre Suma et de la vie du Genji en exil. Sano signale que cette image, qu'on retrouve dans une pièce de nō Suma Genji, fait partie des icônes composant les paysages qui se sont formées à l'époque médiévale inspirées du Roman du Genji. Signalons que le mot, "jeune cerisier » est également catalogué comme une des expressions clés pour composer des vers de renga inspirés du roman. Ainsi, ces études sur la réception du Roman du Genji nous ouvrent vers la recherche interdisciplinaire autour des objets, qui jouent des rôles actifs dans l'organisation "narrative " de genres différents.

7 Anne Bayard-Sakai et Katsumi Fujiwara traitent de front la question de la lecture ou plus exactement de la relecture. Ils la posent par rapport à la construction narrative de l'ensemble, mais de manière diamétralement opposée. Bayard-Sakai s'interroge sur la possibilité de la relecture qui réussit à identifier des microfictions formant des "kystes", selon sa propre expression, dans le roman: des épisodes qui produisent des nouds et qui résistent à être intégrés dans l'architecture narrative en tant qu'éléments composants, même après la relecture qui nous révèle le dessein de l'auteur. Fujiwara de son côté propose dans son article "L'histoire de Kaoru et de la demoiselle Ukifune: ironie et opacité" une relecture attentive à la logique de la construction narrative afin de détecter - c'est là, l'originalité de sa démarche - un épisode virtuel, dont la narration réclame l'existence, mais que l'auteur n'a pas écrit - semble vouloir dire Fujiwara - pour la même raison. Concrètement, il identifie une période de trois mois qui séparent deux chapitres consacrés au destin ironique des deux amants Kaoru, fils adultérin du Genji, et Ukifune, sa protégée qui le trahit, séduite par un prince, puis tente de se suicider. Fujiwara signale les passages que l'auteur a discrètement aménagés de façon à renvoyer à un texte virtuel qui aurait dû représenter une période de bonheur vécue par les amants. Cette remarque fait écho à un chapitre de Roland Barthes par Roland Barthes, intitulé "Opacité et transparence ", titre que nous avons emprunté pour le colloque: 
[...] au terme final (utopique), il y a la transparence : le sentiment tendre, le vœu, le soupir, le désir d'un repos, comme si la consistance de l'interlocution sociale pouvait un jour s'éclaircir, s'alléger, s'ajourer jusqu'à l'invisibilité ${ }^{1}$. appelle wabun 和文, ce « texte en kana », composé en japonais à une époque où la chose écrite l'était encore d'abord en chinois's . Ce n'est pas une simple question de lexique. C'est surtout parce que, depuis, s'est profondément déposée en nous une strate de mots chinois devenus les instruments de notre pensée, une épaisse strate de mots étrangers importés en particulier depuis l'époque moderne. Quelle était la situation à l'époque de Heian pour l'auteur du Roman du Genji ? Il est communément admis qu'elle était sans aucun doute capable de lire les textes en pur chinois. Or, on estime aujourd'hui que le style du Roman du Genji est de toute évidence une des plus belles réussites du wabun. Comment est-ce possible quand on sait qu'une petite centaine d'années seulement s'est écoulée depuis le moment où l'on s'est essayé à écrire et exprimer ses émotions en langue japonaise que l'on notait alors par des kana! En d'autres termes, quelle interaction entre sentiments et mots a pu produire un tel résultat?

11 Moi qui depuis longtemps, cherche à percer ce mystère et à en comprendre les raisons et les secrets, j'ai trouvé une réponse claire dans l'ouvrage Le Roman du Genji : Opacité et transparence - scènes, langage poétique, temps et narration -. J'ai été tout particulièrement intéressé par les articles réunis dans la deuxième session La narration et le langage poétique, dans la mesure où ils sont directement en lien avec mes interrogations. Le Roman du Genji est une œuvre en wabun, c'est à dire en prose. Par opposition, le waka est de la poésie. Malgré cela, ou justement à cause de cela, je pense que ce mode d'expression langagière qu'est le waka a constitué le socle sur lequel s'est bâti ce monde du wabun qu'est le Roman du Genji.

son article "Les dialogues de La fête aux feuilles d'automne", Jacqueline Pigeot traite des échanges de poèmes entre les personnages, considérés sous l'angle du dialogue. Que les poèmes dans le Roman du Genji jouent le rôle d'une conversation entre les protagonistes est en effet généralement admis ; ce qui ne veut pas dire qu'à l'époque de Heian les gens conversaient dans la réalité par le truchement d'un langage inhabituel fait de l'alternance de segments de cinq et sept syllabes. Et donc, le surgissement d'une forme poétique comme le waka au milieu d'un texte en prose, venant se substituer au discours que l'on attendrait des personnages, est la meilleure preuve que l'auteur charge le poème d'exprimer les pensées autrement que ne le ferait de la prose. 
13 Pigeot étudie sous divers angle l'expression poétique, dans le chapitre « Momiji no ga 紅葉賀 (La fête aux feuillages d'automne)». Le poème que je vais reprendre ici, revêt sans doute une importance particulière parmi les poèmes du dit chapitre.

\begin{tabular}{|l|l|}
\hline Sode nururu & Lorsque je m'avise \\
tsuyu no yukari to & qu'il est parent de la rosée \\
omohu ni mo & qui mouille mes manches \\
naho utomarenu & plus fort est mon déplaisir \\
& / il ne peut me déplaire \\
yamato nadeshiko & œillet du Yamato \\
\hline
\end{tabular}

14 Le poème est composé par Fujitsubo, épouse de l'empereur, qui vient de donner naissance à un enfant, fruit de sa relation avec le Prince Genji, fils de ce même empereur. Tel était son destin. Et voilà ce que dit Pigeot à propos de ce poème (p. 130) :

Selon la manière dont on coupe le poème, l'auxiliaire $-n u$, dans la forme verbale utomarenu, peut être compris soit comme l'auxiliaire d'accompli -nu à la forme conclusive (le sens est alors :»je me sens éloignée de cet enfant »), soit comme l'auxiliaire de négation - $z$ à la forme déterminante ( je ne puis me sentir éloignée de cet enfant »). Mais je me demande si choisir entre l'une ou l'autre de ces deux interprétations n'interdit pas de rendre compte de la complexité des sentiments exprimés, n'appauvrit pas le poème. En effet, pour Fujitsubo, l'enfant est à la fois celui de l'homme qu'elle aime plus que tout, donc un enfant qui lui est particulièrement cher, et aussi le reproche vivant de sa faute, donc un être dont elle se sent éloignée, qu'elle rejette. [...] Je pense que seul le poème peut traduire, avec une telle ambiguïté, une telle contradiction dans ce que ressent le personnage.

Auparavant (p.129), Pigeot dit que « la fonction du poème est d'exprimer ce qu'il y a de plus profond en soi », ce qui inclut naturellement les contradictions dans ce que peut ressentir un personnage.

Pour les deux protagonistes qui procèdent à un échange de poèmes, ceux-ci présentent des aspects différents appartenant à trois niveaux: a) le sens porté par les formes langagières, b) le sens que veut y inclure le personnage émetteur, c) le sens reçu par le personnage récepteur; et dans certains cas, ces trois aspects ne coïncident pas forcément. Mais il y a encore une dernière phase qui est celle où s'accomplit finalement l'acte : d) la réaction du destinataire. Le poème qui nous intéresse provoque les pleurs du Genji : » Son cœur tressauta et dans l'excès de sa joie, il répandit des larmes ». Or, il $\mathrm{y}$ a beaucoup de cas opaques, où il est difficile de savoir si la phase $\mathrm{d}$ ) est le reflet direct de la signification transmise au niveau c) ${ }^{3}$.

17 Si l'on en revient au poème, l'interprétation $\mathrm{A}$ « Il ne peut me déplaire » (négation) va dans le sens de l'amour pour son enfant, que le lecteur attend de Fujitsubo en tant que mère. L'interprétation $B$ 《plus fort est mon déplaisir » (perfectif 完了) exprime ce que ressent Fujitsubo devant cet enfant qui lui rappelle sa liaison coupable et risque d'entraîner de la part du lecteur une extrême sévérité due au concept de mère qu'il porte lui-même dans son imagination. De plus : si l'on observe l'image de Fujitsubo dans la suite de la scène, elle apparaît pénétrée d'un amour profond pour son enfant et donc on est plus proche de l'interprétation A «il ne peut me déplaire ». Mais justement, l'interprétation $\mathrm{B}$ "plus grand est mon déplaisir », qui correspond à l'image de Fujitsubo telle qu'elle va se développer dans la suite du roman peut entraîner l'adhésion de lecteurs encore plus nombreux. Le sens B acquiert de la profondeur, dans la mesure où il cache en fait le sens A. À l'inverse, il est aussi évident que le sens A «il 
ne peut me déplaire » recouvre le sens B. C'est ce qui rend possible la poursuite du récit décrivant les relations tendues entre le Genji et Fujitsubo, dans le chapitre "L'arbre sacré » : les nouvelles tentatives du Genji auprès de Fujitsubo, sa retraite au monastère Urin-in après qu'il eut été repoussé par elle, puis finalement la décision prise par Fujitsubo de se retirer du monde.

Ce processus de communication : a) forme exprimée - b) intention - c) réception - d) accomplissement d'un acte, est susceptible de se réaliser aussi bien dans un dialogue en prose. Mais l'insertion des waka dans le roman, du fait que leur forme même constitue un moyen d'expression différent de la prose, rend possible une compréhension mutuelle qui transcende les possibilités langagières de celle-ci. Dans le wabun se rencontrent deux sortes de processus d'associations : celles qui se produisent le long de l'axe diachronique, appelons-les "successions utsuriyuki 移り行き», et celles qui se développent dans la dimension synchronique, c'est à dire des «superpositions kasane 重补 $»^{4}$. On peut dire que la " succession » relève de la contiguïté et la "superposition » de l'analogie. Ces processus contextuels d'associations dans les textes en wabun, trouvent leur origine dans un développement de la sensibilité et de l'expression poétiques.

Si on considère le poème de Fujitsubo comme faisant partie d'une conversation, d'un dialogue, il assume, au premier niveau, de façon immédiate, dans la transmission du message, la fonction dévolue à la prose. Dans ce sens, les waka énoncés par les divers personnages ont qualité de prose. Mais, ici, la fonction de la prose est remplie par cette forme traditionnelle qu'est le waka. Autrement dit, les waka insérés dans le roman sont des waka sans en être. Revenons au waka de Fujitsubo. Les associations: sode - tsuyu, yukari - nadeshiko, qui reprennent les termes du poème envoyé par le Genji, se construisent tout à fait naturellement, un mot en appelant un autre. De la même façon l'expression naho utomarenu peut être considérée comme la "superposition » de deux sentiments contradictoires. Dans les waka énoncés par les personnages, et que l'on peut considérer comme une des formes de la narration, les processus d'association par «succession» et «superposition» fondamentalement poétiques, sont employés directement pour créer un dialogue, fonction que l'on attend de la prose. Et c'est justement ce qui leur permet d'atteindre un maximum d'efficacité. Le lecteur intègre comme tout à fait naturels ces processus d'association que la langue rend possibles, grâce à la culture poétique développée par le waka, et vibre à l'unisson.

Cependant ils ne s'effectuent pas tout à fait librement. Et même il arrive qu'ils ne peuvent se réaliser alors même qu'on le souhaiterait. On peut y voir un moyen d'exprimer sa pensée que permet l'expression en wabun. Les articles de Hijikata Yōichi et de Terada Sumie, présentés lors de la deuxième session La narration et le langage poétique, éclairent chacun à leur façon la signification du dispositif narratif particulier au wabun dans le Roman du Genji.

Le sujet développé par Hijikata dans son article «Le Roman du Genji et le langage de la communauté poétique» concerne les caractéristiques de la culture linguistique et littéraire de cette époque et la façon d'accéder à son dépositaire, ce qu'il appelle la 《 communauté poétique waka kyōdōtai 和歌共同体». Hijikata s'appuie sur un exemple tiré du chapitre "Kashiwagi 柏木 (Le chêne) », du nom du personnage éponyme. Dans le passage cité, ce personnage exprime, en un long discours intérieur, son rêve, sa désillusion, sa liaison coupable découverte, et sa résolution de la racheter par la mort. Voici ce que dit Hijikata à ce propos (p. 117) : 
Cette déploration et ces regrets, loin d'être pris comme l'expression de sentiments personnels, sont ressentis par les gens de cette époque comme se superposant à un code stéréotypé relevant de l'univers poétique. Et du fait qu'elles sont exposées à des lecteurs partageant les connaissances et la culture du waka, ceux-ci sont d'autant plus fortement incités à s'identifier émotionnellement avec le personnage.

regrets de Kashiwagi relèvent, certes, du sentiment personnel, mais si j'ose affirmer avec insistance cette dimension acquise par le langage poétique, ils ne se limitent pas à cette simple dimension. Hijikata insiste sur la différence de nature entre «l'instance individuelle » (conception occidentale moderne du langage) d'une part, et l'individu et son expression langagière dans le Roman $d u$ Genji d'autre part. Dans le Japon ancien, l'individu, le «je » se construit habituellement dans une relation communautaire, le "nous ». Ce qui veut dire, non pas que les frontières entre moi et l'autre sont floues, mais que le moi se réalise dans la "superposition » avec l'autre. Il est donc tout à fait normal que le waka, qu'on compose à partir de cette superposition des sujets, ne parvienne pas à déterminer tout seul sa signification et que, le plus souvent, son interprétation soit si dépendante du contexte situationnel. Pensée d'un individu ou expression de sentiment, plus on cherche à l'exposer clairement, plus on a besoin de faire appel à l'expression poétique traditionnelle, et c'est dans ce processus même que le sens parvient enfin à se fixer. Telle est la nature du waka.

Cette conception du langage peut paraître extrêmement paradoxale du point de vue de la mentalité moderne. Une réalisation langagière employée dans de telles conditions brouille le fondement existentiel de l'individu (ou si on se réfère au titre de cet ouvrage, le rend "opaque »); son réexamen s'impose, dans une confrontation avec le concept de » transparence » porté par la recherche moderne de la clarté. C'est le même problème que la difficulté à trouver facilement dans les romans japonais anciens « le point de vue de l'omniscient ». Le fondement de la communauté poétique, ce qui la rend possible, est la question essentielle liée à l'attitude de l'individu face au langage et à sa vision du monde.

L'article de Terada Sumie «Le monde et son double: le brouillard dans le Roman du Genji » apporte des éléments précieux pour appréhender ce que serait cette conception du langage au Japon à l'époque ancienne. À partir de l'épisode de Rokujō, Terada montre que le brouillard n'est en aucun cas un simple élément de décor secondaire par rapport à l'intrigue, ce qu'elle appelle la «construction narrative", qui serait, elle, principale (p. 84).

Et le brouillard convoqué par elle, fait émerger à son tour un espace appartenant à une dimension du monde différente de celle où se déroule l'histoire des personnages, en l'occurrence, celle de la mort, qui vient côtoyer l'univers des personnages, selon une logique, si j'ose dire, ontologique, en tous cas, outrepassant la logique narrative. (...) Le paysage dans cette « construction narrative » n'est pas un simple décor, sa fonction ne s'arrête pas non plus à être une caisse de résonance des sentiments. (...) C'est un lieu dynamique où émerge par-derrière notre monde, un autre espace qui dépasse notre entendement.

Pour Terada, le brouillard n'est pas une "caisse de résonance ", ce que d'autres ont exprimé par « la concordance entre le paysage et les sentiments keijōicchi 景情一致》, où la description de la nature a pour fonction de décrire l'état intérieur des personnages. Elle fait une autre lecture : il est une voie de communication qui ouvre vers un autre espace. Autrement dit, il relie deux espaces, dont l'un échappe à un ordre préétabli, et donc le lieu où apparaît quelque chose de neuf.

Cipango, $18 \mid 201$ 

deux espaces sont reliés, disons plutôt qu'ils sont en contact par contiguïté. Mais, justement pour cette raison, le langage permet de créer une image nouvelle, différente de celle que chacun de ces espaces évoquait séparément. C'est exactement là que se révèle l'extraordinaire capacité d'expression propre au wabun. Si je le résume avec mes propres termes: "Rokujō convoque le brouillard, le brouillard convoque la mort». Mais ce que je trouve le plus intéressant, ce sont les remarques suivantes: «... le brouillard signale une structure parallèle, la présence d'un monde redoublant le monde normal. » "Soulignons que ce dispositif est entièrement déconnecté de la progression narrative » (p. 85).

Rokujō et la mort sont parallèles dans l'univers du roman. Le processus d'association par » succession » dans le wabun, fait découvrir au lecteur, en douceur, des niveaux de correspondances. Ce qui ne signifie pas que la différence de nature entre les termes de cette association ne soit pas perçue consciemment, qu'elle n'apparaisse pas au premier plan. C'est plutôt que l'on doit se montrer encore plus attentif au fait qu'existe entre eux cette évidente différence de nature, ou selon les termes de Terada, qu'ils se déclenchent indépendamment l'un de l'autre. Différence de nature qui remet en question le point de vue sage et modéré que l'on avait jusqu'à maintenant sur le paysage dans le roman, comme anodin et secondaire. Le titre même Le monde et son double nous invite à réexaminer le rôle du paysage dans le Roman du Genji.

Concernant la construction de l'espace dans le Roman du Genji, la question des "scènes » est un des points qui surgissent inévitablement. L'article de Daniel Struve présenté dans la première session (Scènes, regards, espace théâtral) porte sur les scènes de « vision à la dérobée kaimami 坦間見 ", décrites à plusieurs reprises dans le roman («Vision à la dérobée : un lieu commun et ses variations »). Ce qui m'a profondément intéressé c'est la remarque concernant les points de vue que construit la narration de ces scènes (p. 11).

Dans la scène de vision à la dérobée, le lecteur est invité à s'identifier au personnage. Il voit la scène qui se déploie devant celui-ci, accède au même spectacle, et partage sa fascination. En même temps, il assiste aussi au spectacle du personnage en train de regarder. La scène de vision à la dérobée est en réalité double.

Ceci se présente comme une remarque à propos de la manière de construire une scène de roman, mais plus concrètement elle concerne la relation entre le lecteur et les personnages. Le lecteur fait sien le regard du personnage et s'identifiant à celui-ci, imagine et ressent le spectacle comme s'il le vivait lui-même. Pour le lecteur c'est sans aucun doute un moment de bonheur comme en procure la lecture d'un roman. Mais Struve indique que : »'est aussi pour le lecteur l'occasion d'un retour critique et d'une prise de conscience » (p. 12).

Ainsi, le principe essentiel des scènes de vision à la dérobée, c'est qu'elles sont contées de l'unique point de vue subjectif du personnage. Il est alors intéressant de noter que ces scènes utilisent, à l'évidence, des dispositifs similaires en un sens à ceux qui président à l'activité de composition poétique des personnages, c'est à dire qui mettent en jeu le discours à la première personne. Lorsqu'un personnage compose un poème, c'est une invite encore plus forte faite au lecteur de s'identifier à lui. On peut même dire que c'est dans la mesure où le lecteur ne fait plus qu'un avec le personnage que le sens du poème lui devient accessible. Ce mouvement est encore plus manifeste dans le

Cipango, 18 | 2011 
cas d'un poème soliloqué composé par un personnage isolé, que dans les échanges de poèmes entre deux personnages. Comme le dit Struve, une scène de vision à la dérobée est « un moment d'intensité particulière » (p.12), mais qui n'est vérifié que lorsque le lecteur réussit cette identification. C'est justement la nature de ces scènes qui fait qu'elles acquièrent inévitablement une double dimension : le lecteur faisant retour sur lui-même, voit s'imposer alors à lui le besoin de porter un regard critique.

31 J'illustrerai mon propos par le cas du poème de Fujitsubo que j'ai repris en tête de cet article. Le lecteur, s'identifiant à Fujitsubo, compose ce poème, mais dans le même temps il porte un regard extérieur sur l'état d'esprit de Fujitsubo à ce moment-là, c'està-dire, en fait, sur lui-même, lecteur. C'est ainsi que s'installe l'ambiguïté, l'interprétation du poème partant alors dans deux directions diamétralement opposées. En d'autres termes, on peut dire que ce qui fait que le roman est roman, c'est cette oscillation entre l'identification et la distanciation. Un roman où cette oscillation se produit trop rarement ne peut qu'engendrer l'ennui. Il ne s'y trouve aucune énigme, et il ne sollicite donc pas le regard extérieur. Il me semble que, cette oscillation se produit lorsqu'une scène de vision à la dérobée ou un waka composé par un personnage est placé au cœur du récit. Ceci parce que l'interprétation que le lecteur va faire de telle scène de vision à la dérobée ou de tel waka dépend du déroulement de l'intrigue, de la façon d'être des personnages, avant et après l'épisode considéré.

Pour étudier les dispositifs romanesques, on ne peut donc éviter de se poser la question du lecteur. C'est ce rôle du lecteur dans la composition du roman que traite l'article d'Anne Bayard-Sakai présenté dans la troisième session « L'anecdote ou la microfiction et son rapport avec le lecteur ".

Si l'on revient vers le processus de lecture et la manière dont le lecteur construit progressivement le sens du texte qu'il lit, on a vu à plusieurs reprises qu'il procède par mémorisation et anticipation. Il est donc toujours situé dans un moment du texte, mais il faut surtout insister sur le fait qu'il s'agit d'un mouvement de rectification perpétuel. (p. 146-147)

Dans ces allers et retours entre les parties et le tout, actes de "mémorisation et d'anticipation " à l'œuvre dans le processus de lecture, où le lecteur est en constante référence aux divers événements décrits dans roman, le lecteur porte un regard extérieur sur sa propre lecture pour parvenir à se fixer sur une interprétation. Il se réfère non seulement aux informations contenues dans le roman, mais aussi au cadre culturel, historique qui en constituent le contexte intellectuel. Caresser le rêve de retrouver ce cadre, c'est ce qui définit la «lecture savante»: plaisir de traquer la signification de l'œuvre, mais qui peut exister aussi fortement dans la «lecture nonsavante». Bayard-Sakai en confie le fondement méthodologique à la "relecture». Celle-ci permet d'approfondir la compréhension du sens du roman. Si le Roman du Genji peut encore être reçu par beaucoup de lecteurs aujourd'hui, c'est grâce à cette étroite relation entre parties et tout que la relecture permet de découvrir.

Cependant, je ne crois pas que l'intérêt actuel pour cette œuvre classique ne repose que là-dessus. La tradition du langage poétique, qui sous-tend le wabun, est sans doute devenue étrangère à l'homme d'aujourd'hui. Je pense que très peu de lecteurs ordinaires ont un accès direct à ce qu'on appelle la "communauté poétique " de l'époque. Croire cet accès possible relève, comme le dit Bayard-Sakai, du «fantasme ». Pourtant il est certain que le wabun inspire au lecteur moderne un sentiment de nostalgie, une impression de familiarité. Une des raisons en est que nous avons hérité des valeurs représentées par la nature qui s'est affirmée à travers le waka classique. Le 
lecteur savant, comme le lecteur non-savant partagent cette sensibilité. Le waka est un genre littéraire où l'on fait prendre en charge ses sentiments par les variations de la nature au fil des quatre saisons. Cette communauté de sensibilité à la nature modélisée dans le waka constitue un élément fondamental de la pratique langagière au Japon.

Dans ce sens, l'article de Takada Hirohiko dans la troisième session, «L'organisation narrative du Roman du Genji : épisodes et formes longues - autour de la résidence de la Sixième avenue ", présente un grand intérêt, en particulier les sections: "Les caractéristiques spatiales de la Résidence de la Sixième avenue » et « Les saisons et les personnages ». La résidence que fait édifier le Genji, désignée sous le nom de « Rokujō (Résidence de la sixième avenue)", réunit quatre quartiers. Chaque quartier correspond à une saison: printemps, été, automne, hiver, et constitue l'espace où demeure une femme qui correspond à la valeur symbolique de cette saison. Takada donne à cette fusion de l'espace et des affaires humaines avec les quatre directions et les quatre saisons la signification suivante :

La combinaison des saisons et des personnages que l'on voit dans la Résidence de la sixième avenue est faite d'une double relation: la saison se transforme de l'intérieur en le personnage, et le personnage se manifeste sous l'aspect de la saison. (p. 169)

Pour préciser la pensée qui sous-tend le monde de la Résidence de la sixième avenue : l'homme ne peut exister en dehors de l'écoulement temporel que sont les saisons et le temps ${ }^{5}$; et même s'il existe une hiérarchie sociale entre les personnages ce n'est qu'un phénomène essentiellement relatif dans le cours du temps. (p. 171-172)

Cette subtile observation sur l'accord entre les personnages et la nature met en lumière de façon évidente un sens du langage tout à fait propre au Roman du Genji, et en même temps concerne le cœur même de l'expression en wabun dont ce roman est l'un des plus beaux aboutissements. Les saisons passent, les hommes aussi. L'homme est soumis à la nature, il ne peut s'opposer au cours du temps qui le transforme. Cet accord harmonieux a trouvé sa réalisation et sa forme dans le waka ancien, en particulier dans le Man.yōshā et dans le Kokinshū, et c'est sur cette même sensibilité poétique traditionnelle que le Roman du Genji fonde non seulement son style, mais aussi sa construction. Qu'il y ait encore aujourd'hui des personnes qui apprécient une telle sensibilité prouve bien qu'en dépit du fait que cette forme d'expression dite wabun ne peut plus être employée de nos jours, sa force vitale reste entière.

Lorsque j'ai lu ces articles, je me suis peut-être laissé quelque peu trop emporter du côté du "poétique ", surtout parce que mon intérêt premier concerne la formation du wabun et la fascination que cette prose continue d'exercer. Je serai reconnaissant si l'on comprend que cette démarche était donc pour moi inévitable.

Dans la synthèse qui clôt le volume, l'un des responsables de cette publication, Fujiwara Katsumi dit ceci : « Le tissu linguistique du Roman du Genji est fait de mots qui portent la symbolique et les images de l'univers du waka» (p. 206). S'impose alors l'évidence du lien profond avec le caractère "opaque » du langage poétique. Cet ouvrage s'intéresse cependant davantage, dit-il, aux autres aspects tels que : «l'opacité du tissu de mots propre au texte du roman », »'opacité de l'autre dans les rapports humains tissés par le langage ", " la nature du texte du roman qui fait que les personnages restent opaques pour le lecteur » (p. 207).

39 Si ces exposés, dans leur diversité, m'ont suggéré de si nombreuses réflexions, c'est que la brûlante interrogation qui suscite toutes ces recherches reste la question essentielle 
et résistante de l'«opacité du langage poétique ». Une même approche s'exprime dans nombre d'articles: le Roman du Genji révèle un haut degré de perfection dans la construction de chaque scène et acquiert de ce fait une densité qui le fait d'autant plus apprécier ; mais en même temps il a toutes les qualités d'un long roman montrant une grande profondeur dans sa construction thématique, du fait que les scènes $y$ sont reliées entre elles par un réseau serré de correspondances. Un des éléments qui préside à cette organisation et que j'ai présenté comme central, c'est ce mode de pensée qui trouve son origine dans l'expression poétique : le processus d'association qui se réalise par «succession » ou «superposition ». Peut-être ce présent compte-rendu de lecture s'est-il construit par « succession » selon le mode associatif de la « superposition » avec mes propres problématiques. J'ose espérer que mes choix terminologiques n'ont pas rendu mon texte incompréhensible, pour ne pas dire « opaque »...

\section{NOTES}

1. Coll. «Écrivain de toujours », Paris, Seuil, 1975, p. 141.

2. (NDT) Le terme wabun existe en opposition à celui de kanbun. Le terme kanbun désigne une expression en prose pratiquée au Japon en utilisant uniquement des mots sino-japonais dans une organisation syntaxique soit purement chinoise soit mêlée d'éléments japonais. À une époque où écrire signifiait "écrire en chinois » wabun désigne une expression en prose utilisant quasi exclusivement des mots japonais selon la syntaxe propre à cette langue. Le kanbun s'écrivant en kanji, et le wabun très majoritairement en kana, on rapproche souvent les deux réalités : la langue et sa notation.

3. Matsui Kenji 松井健児, «Zōtōka no hōhō 贈答歌の方法》 (Le procédé des échanges de poèmes), dans Genji monogatari no seikatsu sekai 源氏物語の生活世界 (La vie dans le Roman du Genji), Tōkyō, Kanrin shobō, 2000.

4. Matsui Kenji, «Fūkei wabun no keisei - Genji monogatari no kūkan no seiritsu 風景和文の形 成 - 源氏物語の空間の成立》 (Les descriptions de paysages - La construction de l'espace dans le Roman du Genji), dans Haruo Shirane ハルオシラネ, Fujii Sadakazu 藤井貞和, Matsui Kenji, Nihon bungaku kara no hihyō riron 日本文学からの批評理論 (Théorie critique élaborée du point de vue de la littérature japonaise), Tōkyō, Kasama shoin, 2009. Matsui Kenji, « Fūkei wabun no risō Genji monogatari no shunjū no genkei 風景和文の理想 - 源氏物語の春秋の匀景 》 (L'idéal de la prose descriptive - Les paysages insaisissables des saisons dans le Roman du Genji) dans Imanichi Yūichirō 今西裕一郎, Murobushi Shinsuke 室伏信助, Têma de yomu Genji monogatari ron テーマで 読む源氏物語論 (Pour une lecture thématique du Roman du Genji), vol.3, Tōkyō, Bensei shuppan, 2008. Matsui Kenji, «Fūkei wabun no ryōiki - Genji monogatari no engi suru kūkan 風 景和文の領域一源氏物語の演技する空間》 (Le territoire de la prose descriptive - L'espace comme lieu de mise en scène dans le Roman du Genji), dans Kojima Naoko 小嶋菜温子, Watanabe Yasuaki 渡部泰明, Genji monogatari to waka 源氏物語と和歌 (Le Roman du Genji et le waka), Seikansha, 2008.

5. (NDT) Organisations cyclique et linéaire du temps. 
INDEX

Thèmes : littérature

Keywords : Waburn, waka, Genji monogatari, Heian, monogatari, Novel, Poetry, Language, Literature, Murasaki Shikibu (v. 973-v. 1014 ou 1025), Tale of Genji

Mots-clés : waburn, waka, Genji monogatari, Dit du Genji, roman, poésie, langage, monogatari, Murasaki Shikibu (v. 973-v. 1014 ou 1025)

Index chronologique : Heian 